\title{
Magnetic fields in the Universe and prospects for future probes
}

\section{Philipp Kronberg ${ }^{1}$}

Affiliation, University of Toronto/Los Alamos National Laboratory

Address, Canada/ USA

E-mail: kronberg@physics.utoronto.ca

I describe the latest state of our knowledge of intergalactic magnetic fields, including brief mention of the experimental and observational techniques. A forward look to what the next generation of radio instruments could achieve, as indicated by these current state-of-the-art measurements, suggests some exciting new discoveries that further exploration of the extragalactic radio universe will produce. I also discuss some physical principles that can explain how intergalactic magnetic fields are generated and spread from galaxies into intergalactic space. The IGM may be much more magnetically energized that we previously thought.

\footnotetext{
$1 \quad$ Speaker: Philipp P. Kronberg
} 


\section{Introduction}

In this brief contribution I review some highlights of the measurements, or deduction of magnetic fields in diffuse media, beginning locally with the Milky Way disk, and proceeding to galaxy halos, galaxy clusters, and beyond, up to the largest identifiable structures in the Universe. My description gives particular emphasis to work done by my students, postdocs, other colleagues, and myself, and occasionally I call attention to earlier results that have received rather little attention from the international radio astronomy.

\section{A very brief story of the magnetic field in the Milky Way.}

Measurement of the Milky Way's magnetic field strength began in the 1940's, and the first results came from investigations and analysis not of cosmic radio emission, but of cosmic rays. By the late 1940's cosmic ray nuclei had been found to arrive at the Earth with energies up to $\sim 10^{16} \mathrm{eV}$. Since the CR sources were believed to originate in the Galaxy, e.g. in supernovae and supernova remnants is was remarkable that their arrival directions did not correlate with the plane of the Milky Way. Indeed, up to the highest energies, the arrival directions were found to be isotropic!

This fact was noted by A.Schlüter and Ludwig Biermann (1950), who pointed out that it was an intriguing result. Strange, they argued, because if the CR's were really of universal (extragalactic) origin as implied by their isotropy, the energy content of intergalactic space is implausibly large. This forced the conclusion that the CR's were somehow confined to, and scattered within the Galactic disk - whose thickness was known, and even deducible from visual observations. The only confinement mechanism, Schlüter and Biermann argued, was an interstellar magnetic field, $B_{\text {i.s. }}$ that must permeate the Milky Way disk. In order to produce the observed degree of CR isotropy up to $\approx 10^{16} \mathrm{eV}$, they estimated an interstellar magnetic field strength of $5.2 \pm$ $0.3 \mu \mathrm{G}$. Enrico Fermi (1949) applied similar arguments to arrive at essentially the same conclusion.

This result, which involved no conventional astronomical instrument (i.e. radio or optical telescope), has stood the test of nearly 60 years, and it is remarkably similar to the current consensus value of the interstellar magnetic field strength.

Some of the first astronomical measurements of $B_{\text {i.s }}$ began with attempts by $\mathrm{G}$. Verschuur (1968) and R.D. Davies et al. (1968) at Jodrell Bank in the 1960's to achieve the difficult detection of the Zeeman splitting of the $1420.4 \mathrm{MHz}$ hyperfine transition of interstellar neutral Hydrogen. These gave, initially, values in the range $1-2 \mu \mathrm{G}$, and were later extended by others over many years, notably Carl Heiles at U.C. Berkeley.

The ratio of pulsar RM/DM's has also produced estimates of $B_{\mathrm{i} . s}$, beginning with the first such estimates at Jodrell Bank by A.G. Lyne and F.G. Smith. Pulsar RM/DM measurements give a line of sight average $B_{\text {i.s }}$ due to magnetic field fluctuations along the sightline. Pulsar observations are treated in more detail elsewhere in this conference.

Interstellar linear polarization of starlight was detected by W.A. Hiltner from measurements of the optical polarization of starlight, and subsequently of the Galactic continuum radio emission by Wielebinski, Shakeshaft \& Pauliny-Toth (1962). These 
observations provided early evidence for a significant ordered component of the interstellar magnetic field.

The larger scale Milky Way magnetic field structure using extragalactic radio sources was first investigated by R.D. Davies (1967) and colleagues in Australia. This was followed by the work of Simard-Normandin and Kronberg (1980), who compared RM observations against large scale field models of the Galaxy. Most recently, work by Han and colleagues (this meeting) and Brown and colleagues have further refined models of the ordered $B$ component over the Galactic disk.

\section{Magnetic fields in clusters of galaxies}

It was perhaps the relatively much lower density of the intracluster medium (ICM) that initially discouraged searches for galaxy cluster magnetic fields, the assumption being that ICM fields should be much lower than those in galaxy disks based on their $10-100$ times lower gas densities.

The question of the ICM physics and magnetic field strength was first investigated in some detail by Dennison (1980). He produced estimates in the range $2-$ $10 \mu \mathrm{G}$ for the intracluster medium. Later, Kim et al (1990) analysed the Coma cluster with targeted RM measurements to deduce that Coma had field strengths of order $2 \mu \mathrm{G}$ (scaled to $\mathrm{H}_{0}=75$ ). Now, with better data, it is well established that the ICM of galaxy clusters is magnetized at levels of a few $\mu \mathrm{G}$, and the field strength does not appear affected by the presence or absence of a diffuse, cluster - associated synchrotron halo. It is apparent that the intracluster magnetic field reflects an approximate energy equipartition between $|\boldsymbol{B}|$ and $n_{e} k \mathrm{~T}$ of the hot ICM thermal gas rather than just $n_{e}$.

A current question, not yet well answered, is how the cluster magnetic field "matches" to the magnetic field in the surrounding intergalactic medium (IGM). Attempts to detect $|B|$ in the next hierarchical scale, galaxy filaments, is discussed next.

\section{Expectations for central Black Hole-produced intergalactic magnetic fields}

The enormous energy release from the massive central black holes $(\mathrm{BH})$ of large galaxies is directed via a jet into the surrounding intergalactic medium. At his point we ignore cluster-embedded radio galaxies, whose energy release may be retained for a long time in the host cluster ICM. Letting $\eta$ be the efficiency with which the BH releases magnetic energy into the IGM, we can do a simple calculation.

First the "smoothed-out" BH density can be calculated from estimates of central BH masses derived from galaxy bulge velocity dispersions (e.g. Tremaine et al. 2002). Combining this with the space density of galaxies bearing BH's in the range $10^{6}-10^{9}$ $\mathrm{M}_{\odot}$ gives a space-averaged $\mathrm{BH}$ mass density of

$$
<\rho_{B H}>\approx 2 \times 10^{5} M_{\odot} / M p c^{3}
$$


We can express the energy "available" to be released as a fraction, $\eta$, of the infall energy to a reference radius which we adopt as the BH's Schwarzschild radius, $R_{\mathrm{S}}$. Then

$$
M_{B H} c^{2}=1.8 \times 10^{62} \frac{M_{B H}}{10^{8} M_{\odot}} \operatorname{ergs}
$$

Now with some necessary normalisations, and assuming that half the relativistic gas energy is magnetic, we can calculate the average magnetic energy density that central BH's inject into intergalactic space - which we assume to be the filaments rather than the voids of large scale structure (LSS).

$$
\varepsilon_{\mathrm{B}}=1.36 \times 10^{-15}\left(\frac{\eta_{\mathrm{B}}}{0.1}\right) \times\left(\frac{f_{\mathrm{RG}}}{0.1}\right) \times\left(\frac{f_{\mathrm{FIL}}^{V O L}}{0.1}\right)^{-1} \times\left(\frac{M_{\mathrm{BH}}}{10^{8} M_{\odot}}\right) \mathrm{erg} \mathrm{cm}^{-3},
$$

so that

$$
B_{\mathrm{IG}}^{\mathrm{BH}}=\sqrt{8 \pi \varepsilon_{\mathrm{B}}}=1.8 \times 10^{-7} \mathrm{G} .
$$

$\eta_{\mathrm{B}}$ is the efficiency of conversion of gravitational to magnetic energy, here adopted as $10 \%, f_{\mathrm{RG}}$ is the fraction of large galaxies over a Hubble time that have been a jet-lobe radio source and $f^{\mathrm{VOL}}$ FIL is the fraction of all intergalactic space occupied by galaxy filaments and sheets. Variations by a factor of a few in each of the adopted normalization factors in (3) will, in combination, not likely have a large effect on $B_{\mathrm{IG}}^{\mathrm{BH}}$

These numbers suggest that magnetic fields at the level of a few times $10^{-7} \mathrm{G}$ might be detectable over scales of a few Mpc in the galaxy filament zones of LSS. They are potentially within reach of observational detection, either by Faraday rotation or perhaps by faint synchrotron radiation.

\section{Search for a detectable magnetic field in galaxy filaments in cosmological large scale structure (LSS)}

I now briefly describe a first attempt at a Faraday rotation detection of intergalactic magnetic fields at these levels, partly with the purpose of giving a "feel" for what could be done with a future SKA combined with sensitive deep galaxy surveys with spectroscopic redshifts.

A related way of posing the question of expectations for $B_{\text {IG }}$ is to proceed from the progression of magnetic field strengths from the galaxy discs to (comparable) values in cluster ICM's. This naturally leads us to ask if the next level of cosmic system scale, filaments or chains of galaxies, possess a magnetized environment that that scales in some physically sensible way. The previous section presented one such calculation of "expectation".

The angular density of accurate Faraday rotation measures on the Galactic sky is now just good enough to test whether some local Universe galaxy filaments add an "RM signal" to radio sources behind them. In the first investigation of this kind, Xu et al. (2006) used both the CfA and 2MASS galaxy surveys to model galaxy filaments. For the CfA survey, it was possible to tessilate a 3-D model. The 2MASS survey which, unlike the CfA did not have spectroscopic redshifts, can nonetheless define a galaxy column density for the supercluster filaments. Three local universe superclusters were 
investigated; Virgo, Hercules and Perseus-Pisces, by comparing an intergalactic weighted path length (CfA) or galaxy column density (2MASS) with extragalactic source RM's. The latter were "cleaned" of anomalously large RM's to better detect an expected weak RM signal from the supercluster filaments. Of the three superclusters, only Perseus-Pisces, which has the best-delineated chain of galaxies, showed an an interesting RM signal consistent with a filament-associated, IGM field strength of order $0.1 \mu \mathrm{G}$-- just what we might expect from section 4 . Figure 1 illustrates the RM sky omitting the galactic plane zone and the less densely covered southern sky, compared with the a smoothed galaxy column density map. Further details can be found in $\mathrm{Xu}$, Kronberg, Habib \& Dufton (2006).

It is always possible, of course, that a foreground large scale Galactic feature could (perversely) mimic the effect of a galaxy supercluster filament. Looking to the future however these observations provide an interesting, and tantalizing testbed for what will be possible in future with extragalactic RM's for sources of known redshift that much more densely cover the sky.
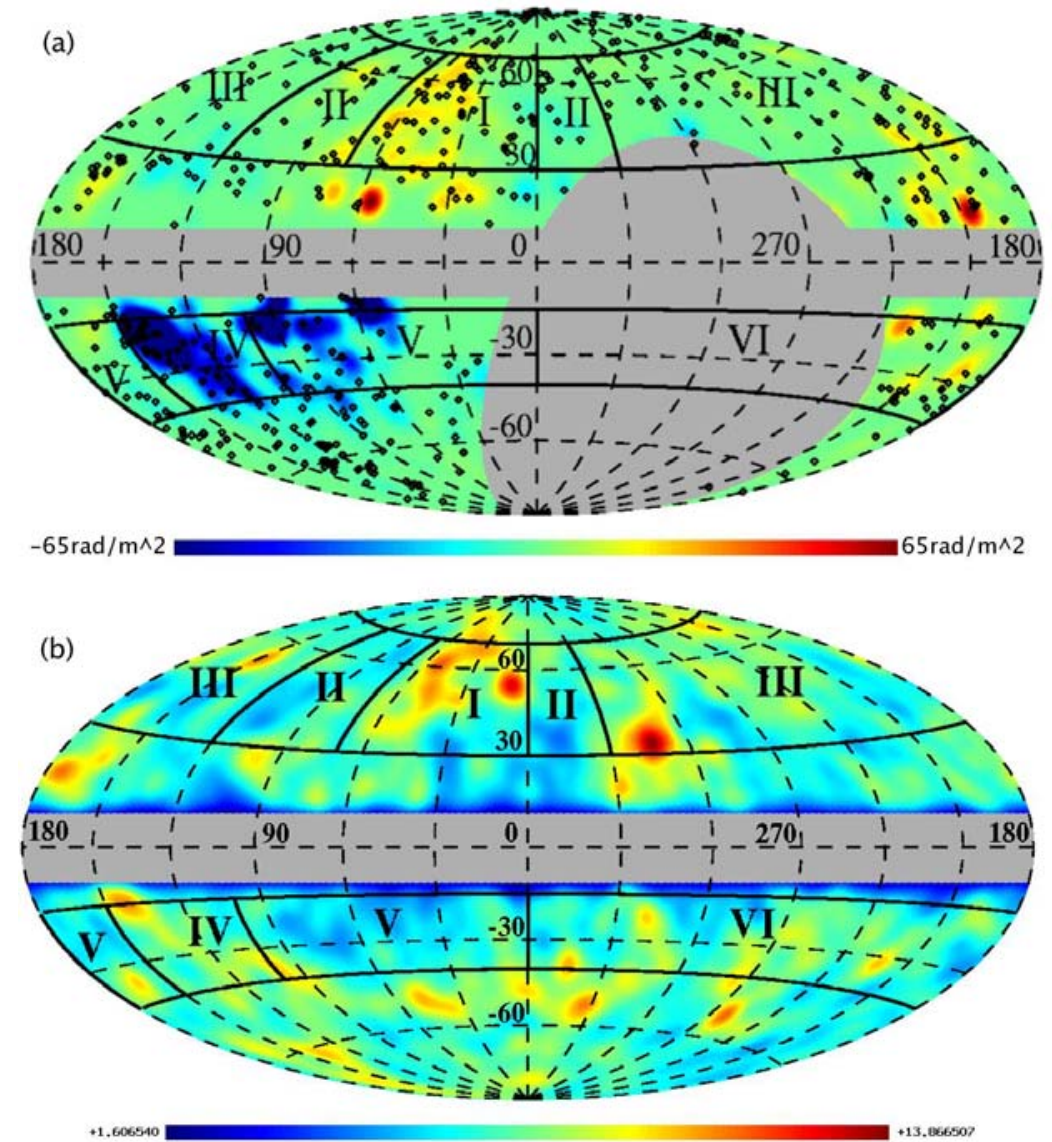

Figure 1: Comparison of a portion of the smoothed RM sky compared with a comparably smoothed, magnitude-limited galaxy distribution from the 2MASS survey.The false colours represent RM (upper) and relative galaxy surface density (lower). (Xu, Kronberg, Habib \& Dufton, ApJ $\underline{637}, 19,2006$.) Source: The Astrophysical Journal 


\section{Large radio sources as calorimeters of $\mathrm{BH}$ energy release}

It is illuminating, as shown by Kronberg et al. (2001), to compare the energy contents of different classes of extended radio sources with each other and with the energy of the original BH "reservoir". In Figure 2, adapted from this paper, the approximate energy contents are plotted against overall source size for two classes of source. (1) those with the largest dimensions, cut off at a lower bound of $\sim 6.5 \mathrm{Mpc}$ in projection, and (2) a sample within $150 \mathrm{kpc}$ of the X-ray centre of a galaxy cluster.

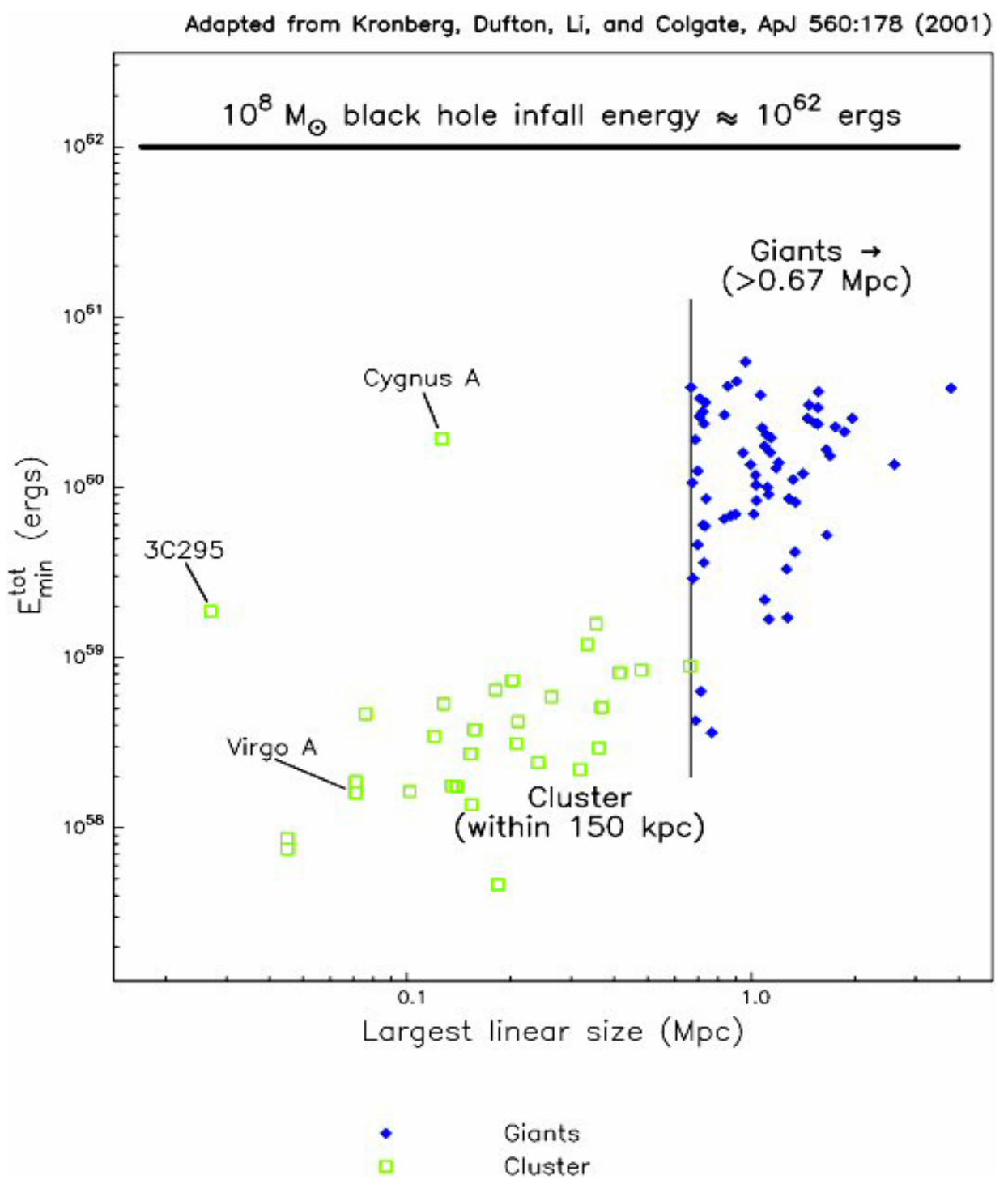

Figure 2: The total energies of (1) giant (blue diamonds), and (2) cluster-embedded radio sources (green boxes) compared, and with the approximate infall energy of a $10^{8} \mathrm{M}_{\odot}$ central black hole.

(Adapted from Kronberg, Dufton, Li, \& Colgate ApJ 2001).

The upper envelope of the giant sources energies represents an energy level within only $\sim 1 \frac{1}{2}$ orders of magnitude of the formation energy (Equation (2)) of a 
$10^{8} \mathrm{M}_{\odot}$ central BH. The sources' internal energy calculation requires assumptions about the energy fraction $(k)$ in the invisible relativistic protons, and the internal volume filling factor $(\varphi)$ of the synchrotron radiating plasma, which could reduce the energy estimates in Fig 2. These were adopted as $\boldsymbol{k}=100$ and $\boldsymbol{\varphi}=0.1$ in the above plot, so that lower values of either would reduce the energy estimates somewhat, but not drastically. On the other side of this energy ledger, the giant source radio lobes will already have lost energy due to some $P d V$ work done by the time they reach their observed sizes. In addition to the $P d V$ work, an upwards correction of the points in Fig. 2 should also be made for diffusion and radiation losses.

If the effective final infall radius $R$ at the $\mathrm{BH}$ is larger than $R_{S}$, the Schwarzschild radius, the upper line in Figure 2 would be lower in proportion to $R_{S} / R$. Such a situation is plausible, for example if the gravitational-to-magnetic coupling happens at a few times $R_{S}$ in the $\mathrm{BH}$ accretion disk. That would also reduce the gap with the upper envelope of the giants in Figure 2. The smallness of the gap gives a direct confirmation from radio measurements that the gravitational- to- relativistic plasma energy ratio, $\boldsymbol{\eta}$, i.e. efficiency, is high and it justifies the normalisation $\boldsymbol{\eta}_{\mathrm{B}}=0.1$ used in equation (3).

Finally, we note that those sources in Fig 2 at $\boldsymbol{r}_{c l}<150 \mathrm{kpc}$ have strikingly lower energy contents than their giant radio source counterparts. But when the very substantial $P d V$ work of the cluster-embedded sources is calculated using the measured thermal intracluster hot gas pressure from X-ray images, these energies get upwards-corrected to be comparable with the giant radio sources! For further details the reader is referred to Kronberg, Dufton, Li, and Colgate (2001).

\section{A new, deep search for faint diffuse synchrotron radiation}

A recent, novel combination at $0.4 \mathrm{GHz}$ of the Arecibo radio telescope, and the 7element DRAO Interferometer at Penticton BC has produced the deepest radio image of diffuse emission yet seen at on resolution scales down to 5' (Kronberg, Kothes, Salter

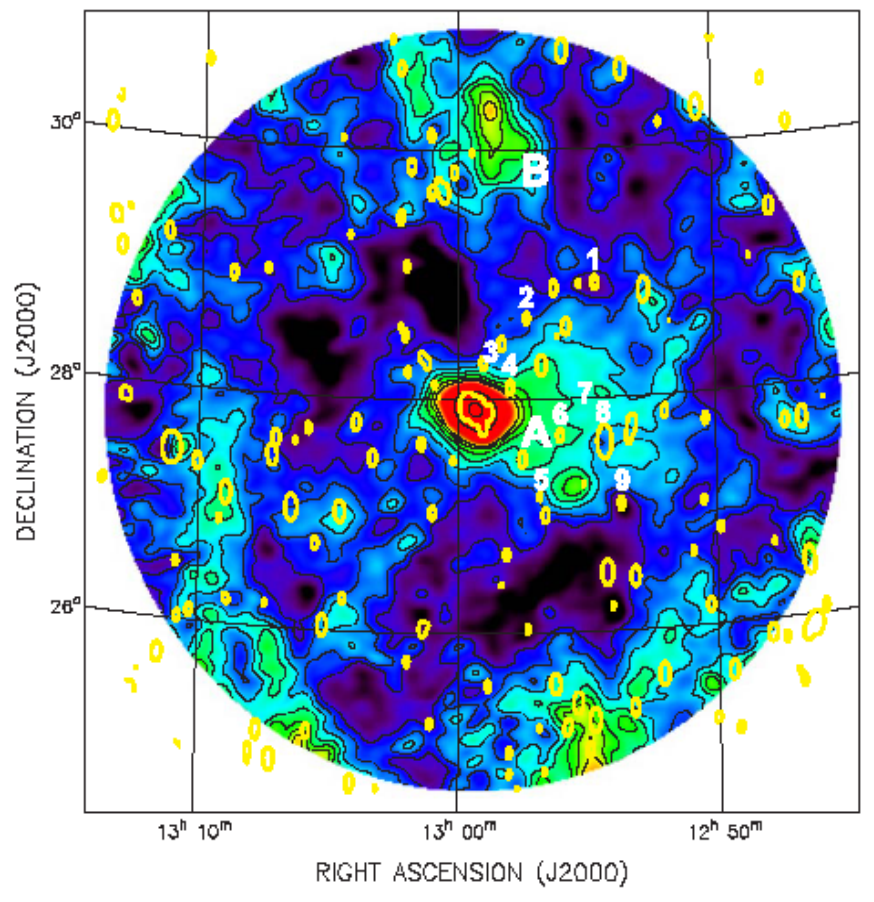

Figure 3: A $0.4 \mathrm{GHz}$ image of an $8^{\circ}$ diameter field near the North Galactic Pole, showing the radio halo of the Coma cluster of galaxies. The image represents a combination of the Arecibo telescope and the DRAO Interferometer. Ellipses representing the brightest removed discrete sources show the $2.5^{\prime} \times 6.5^{\prime}$ half power dimensions of the DRAO beam. For further description see the text, and Kronberg, Kothes, Salter, \& Perillat ApJ 659, 267, 2007. (Source: the Astrophysical Journal) 
and Perillat 2007). The combined image at the Arecibo resolution is reproduced in Figure 3. Locations where the brightest sources were removed are shown by yellow ellipses. Only diffuse emission remains after subtraction of all discrete sources imaged by the DRAO interferometer. Among several newly revealed features is a $2-4 \mathrm{Mpc}$, westward extension of the Coma cluster's radio halo. This zone of intergalactic synchrotron emission has comparable dimensions to giant radio sources discussed above, except that it does not have a single galaxy progenitor. Rather, there are $6-8$ radio galaxies co-spatially associated with this giant diffuse feature. The energy content of this Region A is thus consistent with the aggregate release of magnetic and particle energy from these $6-8$ galaxies. This is the first indication of global energy input from central galaxy nuclei consistent with the calculation in equation (3). A second prominent diffuse feature, $\mathrm{B}$ is $\sim 1^{\circ}$ in extent, and although $1.5 \mathrm{Jy}$ in total flux it has not previously been seen. The image also reveals two deep "holes" that provide a radio window to the outside universe for exploring small scale CMB fluctuations.

\section{Acknowledgements}

I am indebted to the many co-workers and co-authors involved in the results summarized in this brief conference review, and to Rob Beswick for help with the final formatting of this article. I am also grateful for the support of the Natural Sciences and Engineering Research Council of Canada (NSERC), the U.S. Department of Energy (DOE), and a current Distinguished Visiting Scientist appointment at the University of Sydney and CSIRO.

\section{References}

[1] A. Schlüter \& L. Biermann, Zeitschrift. für Naturforschung. $\underline{\mathbf{5 A}}, 237,1950$

[2] E. Fermi, Phys. Rev. $\underline{75}, 1169,1949$

[3] Davies, R. D. Booth, R.S., \& Wilson, A.J., Nature, 220, 1207, 1968

[4] Verschuur, G. L., PRL, 포, 775, 1968

[5] Davies, R.D., IAU Symp. No $\underline{31}, 391,1967$

[6] Wielebinski, R., Shakeshaft, J. R., \& Pauliny-Toth, I. I. K. The Observatory, 2, 158, 1962

[7] Simard-Normandin, M., \& Kronberg, P.P. Astrophys J. 242, 74, 1980.

[8] Dennison, B. Astrophys. 239, L93, 1980

[9] Tremaine, S. D. et al., Astrophys. J. 문, 740, 2002

[10] Kim, K.T., Kronberg, P. P., Dewdney, P. E., \& Landecker, T. L. Astrophys. J. $\underline{355}$, 29, 1990

[11] Xu, Y., Kronberg, P. P., Habib, S., \& Dufton, Q. W. Astrophys. J. $\underline{\mathbf{3 5 5}}$, 29, 2006

[12] Kronberg, P. P., Dufton, Q. W., Li, H., \& Colgate, S. A. Astrophys. J. $\underline{\mathbf{5 6 0}}, 178,2001$

[13] Kronberg, P. P., Kothes, R., Salter, C. J. and Perillat, P. Astrophys. J. $\underline{\mathbf{6 5 9}}$, 267, 2007 